\title{
Right Approximations and Recollements
}

\author{
Xuerong $\mathrm{Fu}^{*}$, Hailou Yao \\ College of Applied Sciences, Beijing University of Technology, Pingle Yuan 100, Beijing, 100124, P. R. China. \\ *Corresponding author. Tel.: +86-18810923976; email: xuerongfu.0098@163.com \\ Manuscript submitted April 17, 2017; accepted June 15, 2017. \\ doi: 10.17706/ijapm.2017.7.3.173-181
}

\begin{abstract}
The purpose of this paper is to investigate how the relative dimension with respect to right approximations behaves on a recollement $(\mathcal{A}, \mathcal{B}, \mathcal{C})$ of abelian categories $\mathcal{A}, \mathcal{B}$ and $\mathcal{C}$. As an application, the relative dimensions with respect to tilting objects of abelian categories involved in a recollement $(\mathcal{A}, \mathcal{B}, \mathcal{C})$ are recovered.
\end{abstract}

Key words: Recollement, right approximation, abelian category.

\section{Introduction}

Thirty-five years ago, Beilinson, Bernstein and Deligne [1] first introduced the recollements of triangulated categories in the construction of perverse sheaves. Now it plays an important role in algebraic geometry, representation theory, polynomial functor theory and ring theory, see for example [1]-[4] and references therein. Psaroudakis and Vitória observed that a recollement whose terms are module categories is equivalent to one induced by an idempotent element and is extensively studied (see [3], [4]).

The relative homological dimension with respect to contravariantly finite subcategories was defined by Dugas [5] in order to generalize some standard theory of projective modules (also see [6]). In 2014, Psaroudakis explicitly investigated how various homological invariants and dimensions of the categories involved in a recollement are related. In particular, he showed that the homological dimensions of $\mathcal{B}$ can be bounded by the homological dimensions of $\mathcal{A}$ and $\mathcal{C}$.

Motivated by the above research mentioned, our main aim of this present paper is to study how the relative dimension with respect to right approximations behaves in a recollement $(\mathcal{A}, \mathcal{B}, \mathcal{C})$ between abelian categories. Throughout, we denote by $\mathbb{N}, K$ and Id the set of nonnegative integers, an algebraic closed field and the identity functor, respectively.

\section{The Relative Homological Dimension with Respect to Right Approximations}

\subsection{Definition}

Let $\mathcal{B}$ be an abelian category [7], the subcategory $\mathfrak{A}$ of $\mathcal{B}$ is said to be contravariantly finite if each object $B$ in $\mathcal{B}$ has a right $\mathfrak{A}$-approximation. In particular, for an object $B$ in $\mathcal{B}$, the morphism $f: X \rightarrow B$ with $X \in \mathfrak{A}$ is called a right $\mathfrak{A}$-approximation of $B$ if $\operatorname{Hom}_{\mathcal{B}}(\mathfrak{A}, X) \rightarrow \operatorname{Hom}_{\mathcal{B}}(\mathfrak{A}, \mathrm{B}) \rightarrow 0$ is exact.

The notion of a left $\mathfrak{A}$-approximation and the notion of a covariantly finite subcategory can be defined dually. When a subcategory $\mathfrak{A}$ of $\mathcal{B}$ is both contravariantly finite and covariantly finite, it is called a 
functorially finite subcategory. For example, the module category $\bmod \Lambda$ over an artin algebra $\Lambda$ has a contravariantly finite subcategory, which is the subcategory consisting of all projective $\Lambda$-modules, denoted by $\operatorname{proj} \Lambda$. For any $K$-coalgebra $C$, let $\operatorname{inj} \Lambda$ be the subcategory consisting of all injective $\operatorname{right} C$ -comodules, which is a covariantly finite subcategory of Comod - $C$. If $C$ is a right semiperfect coalgebra, then Comod - $C$ both has a contravariantly finite subcategory and a covariantly finite subcategory.

From now on, we assume that $\mathfrak{A}$ is always a contravariantly finite subcategory of $\mathcal{B}$. Then we give the following definitions which play an important role in our main results.

\subsection{Definition}

Let $\mathfrak{A} \subseteq \mathcal{B}$ be a contravariantly finite subcategory with abelian category $\mathcal{B}$. An $\mathfrak{A}$ resolution of $M \in \mathfrak{A}$ is the following exact sequence

$$
\cdots \rightarrow T_{n} \rightarrow T_{n-1} \rightarrow \cdots \rightarrow T_{1} \rightarrow T_{0} \rightarrow M \rightarrow 0
$$

with each $T_{i} \in \mathfrak{A}$ such that the induced sequence

$0 \rightarrow \operatorname{Hom}_{\mathcal{B}}\left(T, T_{n}\right) \rightarrow \operatorname{Hom}_{\mathcal{B}}\left(T, T_{n-1}\right) \rightarrow \cdots \rightarrow \operatorname{Hom}_{\mathcal{B}}\left(T, T_{1}\right) \rightarrow \operatorname{Hom}_{\mathcal{B}}\left(T, T_{0}\right) \rightarrow \operatorname{Hom}_{\mathcal{B}}(T, M) \rightarrow 0$

is exact for all $T \in \mathfrak{A}$.

\subsection{Remark}

The methods of constructing an $\mathfrak{A}$-resolution of an object in $\mathcal{B}$ is similar to constructing a projective resolution in [8, Proposition 6.2]. Here, we start by taking a right $\mathfrak{A}$-approximation $f_{0}: T_{0} \rightarrow M$ of $M$ and continue by taking $f_{i}: T_{i} \rightarrow \operatorname{Ker} f_{i-1}$ to be a right $\mathfrak{A}$-approximation for each $i$. Since $\mathfrak{A}$ contains all projective objects of $\mathcal{B}$, it follows that each right $\mathfrak{A}$-approximation is an epimorphism and the resolution preserves exactness.

If $\mathfrak{A} \subseteq \mathcal{B}$ is a contravariantly finite subcategory, then the $\mathfrak{A}$-dimension of $M \in \mathcal{B}$ is defined by $\mathfrak{A}-\operatorname{dim}(M)=\min \left\{n \in \mathbb{N} \mid \Omega_{\mathfrak{A}}^{n+1} M=\operatorname{Ker}_{n}=0\right\}$. If we can't find such $n \in \mathbb{N}$ satisfying $\operatorname{Ker} f_{n}=0$, then we say $\mathfrak{A}-\operatorname{dim}(M)=\infty$.

The $\mathfrak{A}-\operatorname{dimension}$ of $\mathcal{B}$ is defined by $\mathfrak{A}-\operatorname{dim}(\mathcal{B})=\sup \{\mathfrak{A}-\operatorname{dim}(\mathrm{M}) \mid \forall M \in \mathcal{B}\}$

\subsection{Definition}

[4, Definition 2.1] A recollement situation between abelian categories $\mathcal{A}, \mathcal{B}$ and $\mathcal{C}$ is a diagram

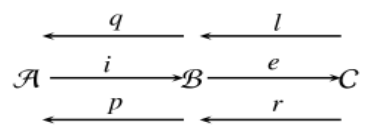

denoted by $(\mathcal{A}, \mathcal{B}, \mathcal{C})$, satisfying the following conditions:

(r1) $(q, i, p)$ and $(l, e, r)$ are adjoint triples;

(r2) the functors $i, l$ and $r$ are fully faithful; 
(r3) $\operatorname{Im} i=$ Kere.

\subsection{Remark}

1) The functors $e: \mathcal{B} \rightarrow \mathcal{C}$ and $i: \mathcal{A} \rightarrow \mathcal{B}$ are exact. Moreover, $q i \simeq \operatorname{Id}_{\mathcal{A}}, \operatorname{Id}_{\mathcal{A}} \simeq p i$, $e r \simeq \operatorname{Id}_{\mathcal{C}}$ and $\operatorname{Id}_{\mathcal{C}} \simeq e l$

2) If the pair $(l, e)$ is an adjoint functor pair and the functor $e$ is exact, then the left adjoint functor $l$ preserves projective objects.

3) If the pair $(e, r)$ is an adjoint functor pair and the functor $r$ is exact, then the left adjoint functor $e$ preserves projective objects.

4) If the pair $(l, e)$ is an adjoint functor pair and the functor $l$ is exact, then the right adjoint functor $e$ preserves injective objects.

5) If the pair $(e, r)$ is an adjoint functor pair and the functor $e$ is exact, then the right adjoint functor $r$ preserves injective objects.

6) For any adjoint functor pair, the left adjoint functor preserves the right exactness and commutes with any direct sums; the right adjoint functor preserves the left exactness and commutes with any direct products, such as for the adjoint pair $(l, e)$, we have that $\operatorname{Add}(l(M))=l \operatorname{Add}(M)$ and $\operatorname{Prod}(e(N))$ $=e(\operatorname{Prod}(N))$.

\subsection{Lemma}

Let $(\mathcal{A}, \mathcal{B}, \mathcal{C})$ be a recollement of abelian categories.

1) If $\mathfrak{A}$ is a contravariantly finite subcategory of $\mathcal{B}$, then $e(\mathfrak{A})$ is a contravariantly finite subcategory of $\mathcal{C}$.

2) If $\mathfrak{A}$ is a covariantly finite subcategory of $\mathcal{B}$, then $e(\mathfrak{A})$ is a covariantly finite subcategory of $\mathcal{C}$.

3) If $\mathfrak{A}$ is a functorially finite subcategory of $\mathcal{B}$, then $e(\mathfrak{A})$ is a functorially finite subcategory of $\mathcal{C}$.

Proof. (1) For any object $C \in \mathcal{C}$, since $\mathfrak{A}$ is a contravariantly finite subcategory of $\mathcal{B}$, it follows that for

$r(\mathrm{C}) \in \mathcal{B}$, there exists a right $\mathfrak{A}$-approximation of $r(\mathrm{C})$, that is, $f: X \rightarrow r(\mathrm{C})$ with $X \in \mathfrak{A}$ such that the sequence

$$
\operatorname{Hom}_{\mathcal{B}}(\mathfrak{A}, X) \rightarrow \operatorname{Hom}_{\mathcal{B}}(\mathfrak{A}, r(C)) \rightarrow 0
$$

is exact in $\mathcal{B}$. Applying the exact functor $e$, we have $e(f): e(X) \rightarrow C$, since $e r \simeq \operatorname{Id}_{\mathcal{C}}$. To show that $e(\mathfrak{A})$ is a contravariantly finite subcategory of $\mathcal{C}$, we need prove that

$$
\operatorname{Hom}_{\mathcal{C}}(e(\mathfrak{A}), e(X)) \rightarrow \operatorname{Hom}_{\mathcal{C}}(e(\mathfrak{A}), C) \rightarrow 0
$$

is exact in $\mathcal{C}$, i.e. we have to show that the morphism $\operatorname{Hom}_{\mathcal{C}}(e(\mathfrak{A}), e(X)) \rightarrow \operatorname{Hom}_{\mathcal{C}}(e(\mathfrak{A}), C)$ is an epimo-rphism. Let $c: e(B) \rightarrow C$ be a morphism in $\mathcal{C}$. Then the following composition of morphisms

$$
B \stackrel{v_{B}}{\longrightarrow} r e(\mathrm{~B}) \stackrel{r(\mathrm{c})}{\longrightarrow} r(\mathrm{C})
$$

belongs to $\operatorname{Hom}_{\mathcal{B}}(B, r(C))$, where $v_{B}$ is the unit of the adjoint pair $(e, r)$. Hence from (2.3) there exists a 
morphism $\kappa \in \operatorname{Hom}_{\mathcal{B}}(B, X)$ such that $\kappa \bullet f=v_{B} \bullet r(\mathrm{c})$. Then $e(\kappa) \bullet e(f)=e\left(v_{B}\right) \bullet \operatorname{er}(\mathrm{c})=\mathrm{c}$, and so the sequence $\operatorname{Hom}_{\mathcal{C}}(e(\mathfrak{A}), e(X)) \rightarrow \operatorname{Hom}_{\mathcal{C}}(e(\mathfrak{A}), C) \rightarrow 0$ is exact in $\mathcal{C}$. Therefore, $e(\mathfrak{A})$ is a contravariantly finite subcategory of $\mathcal{C}$. The assertion (2) is a duality of (1). And (3) follows directly from (1) and (2).

\subsection{Lemma}

Let $(\mathcal{A}, \mathcal{B}, \mathcal{C})$ be a recollement of abelian categories.

1) If $\mathfrak{A}$ is a contravariantly finite subcategory of $\mathcal{B}$, then $q(\mathfrak{A})$ is a contravariantly finite subcategory of $\mathcal{A}$.

2) If $\mathfrak{A}$ is a covariantly finite subcategory of $\mathcal{B}$, then $q(\mathfrak{A})$ is a covariantly finite subcategory of $\mathcal{A}$.

3) If $\mathfrak{A}$ is a functorially finite subcategory of $\mathcal{B}$, then $q(\mathfrak{A})$ is a functorially finite subcategory of $\mathcal{A}$.

Proof. (1) For any object $A \in \mathcal{A}$, since $\mathfrak{A}$ is a contravariantly finite subcategory of $\mathcal{B}$, it follows that for $i(A) \in \mathcal{B}$, there exists a right $\mathfrak{A}$-approximation of $i(A)$, that is, $g: Y \rightarrow i(A)$ with $Y \in \mathfrak{A}$ such that the sequence

$$
\operatorname{Hom}_{\mathcal{B}}(\mathfrak{A}, Y) \rightarrow \operatorname{Hom}_{\mathcal{B}}(\mathfrak{A}, i(A)) \rightarrow 0
$$

is exact in $\mathcal{B}$. Applying the exact functor $q$, we have $q(g): q(Y) \rightarrow A$, since $q i \simeq \operatorname{Id}_{\mathcal{C}}$. To show that $q(\mathfrak{A})$ is a contravariantly finite subcategory of $\mathcal{A}$, it is sufficient to prove that

$$
\operatorname{Hom}_{\mathcal{A}}(q(\mathfrak{A}), q(Y)) \rightarrow \operatorname{Hom}_{\mathcal{A}}(q(\mathfrak{A}), A) \rightarrow 0
$$

$\mathcal{A}$

is exact in . It is equivalent to show that the morphism $\operatorname{Hom}_{\mathcal{A}}(q(\mathfrak{A}), q(g))$ is an epimorphism. Let $c: q(B) \rightarrow A$ be a morphism in $\mathcal{A}$. Then the following composition of morphisms

$$
B \stackrel{\kappa_{B}}{\longrightarrow} i q(B) \stackrel{\mathrm{i}(\mathrm{c})}{\longrightarrow} i(A)
$$

belongs to $\operatorname{Hom}_{\mathcal{B}}(B, i(A))$, where $\kappa_{B}$ is the unit of the adjoint pair $(i, q)$. Hence from (2.4) there exists a morphism $v \in \operatorname{Hom}_{\mathcal{B}}(B, Y)$ such that $v \llbracket g=\kappa_{B} \square i(\mathrm{c})$. Then $q(v) \square q(\mathrm{~g})=q\left(\kappa_{B}\right) \square q i(c)=c$, and so the sequence $\operatorname{Hom}_{\mathcal{A}}(q(\mathfrak{A}), q(Y)) \rightarrow \operatorname{Hom}_{\mathcal{A}}(q(\mathfrak{A}), A) \rightarrow 0$ is exact in $\mathcal{A}$. Therefore, $q(\mathfrak{A})$ is a contravariantly finite subcategory of $\mathcal{A}$. The assertion (2) is a duality of (1). And (3) follows directly from (1) and (2).

Given two algebras $B, C$ and a finite-dimensional $C$ - $B$-bimodule $M$, we can define a triangular matrix

algebra $\Lambda=\left(\begin{array}{cc}B & 0 \\ M & C\end{array}\right)$. The multiplication of $\Lambda$ is given by the matrix product. For more details about its related properties, we refer the readers to [9, Section III2] and [10, Section 1]. It is well known that there is a categorical description for the triangular matrix algebra. Since $\Lambda$ is glued from $B$ and $C$ by the bimodule $M$, the category $\bmod \Lambda$ is closely related to categories $\bmod B$ and $\bmod C$. If $\varepsilon_{B} \in B$ and $\varepsilon_{C} \in C$ are the identities of $B$ and $C$, respectively, then $\varepsilon_{\Lambda}=\varepsilon_{B}+\varepsilon_{C}$ and $B \oplus M=\Lambda \varepsilon_{B} \Lambda, C \oplus M=\Lambda \varepsilon_{C} \Lambda$.

Moreover we also have $B \cong \Lambda / \Lambda \varepsilon_{C} \Lambda$ and $C \cong \Lambda / \Lambda \varepsilon_{B} \Lambda$. It follows from [3, Theorem 5.3] that there 
are two recollements as follows

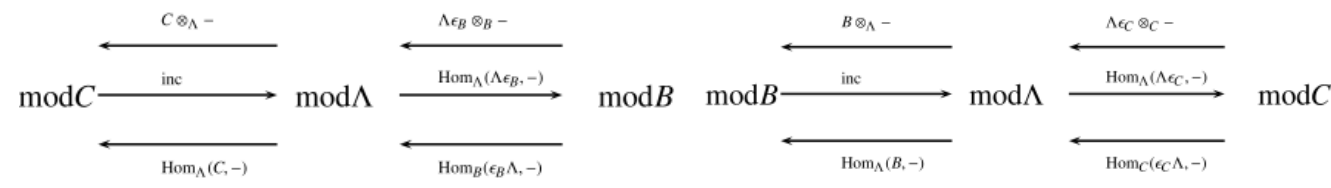

Now we use the Theorem 2.1 of [10] to show a situation of the above lemma.

\subsection{Corollary}

Let $\Lambda=\left(\begin{array}{cc}B & 0 \\ M & C\end{array}\right)$ be a triangular matrix algebra, $U$ a full subcategory of $\bmod B, V$ a full subcategory of $\bmod C$ and let $W=\{(X, Y, f) \mid X \in U, Y \in V\}$. Then we have

1) $W$ is contravariantly finite in $\bmod \Lambda$ if and only if $U$ is contravariantly finite in $\bmod B$ and $V$ is contravariantly finite in $\bmod C$;

2) $W$ is covariantly finite in $\bmod \Lambda$ if and only if $U$ is covariantly finite in $\bmod B$ and $V$ is covariantly finite in $\bmod C$;

3) $W$ is functorially finite in $\bmod \Lambda$ if and only if $U$ is functorially finite in $\bmod B$ and $V$ is functorially finite in $\bmod C$.

\section{Main Results}

Now, we have prepared all the ingredients to state our main results in this paper.

\subsection{Lemma}

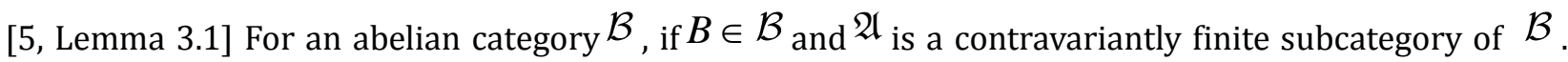
Then the following statements are equivalent:

1) $\mathfrak{A}-\operatorname{dim}(M) \leq k$.

2) the minimal $\mathfrak{A}$-resolution of $M$ has length at most $k$.

3) $M$ has an $\mathfrak{A}$-resolution $0 \rightarrow T_{k} \rightarrow T_{k-1} \rightarrow \cdots \rightarrow T_{1} \rightarrow T_{0} \rightarrow M \rightarrow 0$,

4) $\operatorname{Ext}_{\mathcal{B}}^{i}(M, Y)=0$ for all $i>k$ and all objects $Y \in \mathfrak{A}$.

\subsection{Theorem}

Let $(\mathcal{A}, \mathcal{B}, \mathcal{C})$ be a recollement of abelian categories with a contravariantly finite subcategory $\mathfrak{A} \subseteq \mathcal{B}$. If $\mathfrak{A}-\operatorname{dim}(\mathcal{B}) \leq k$ and $r e(\mathfrak{A}) \subseteq \mathfrak{A}, i q(\mathfrak{A}) \subseteq \mathfrak{A}$, then we have

1) $e(\mathfrak{A})-\operatorname{dim}(\mathcal{C}) \leq k$,

2) if the functor $q: \mathcal{B} \rightarrow \mathcal{A}$ is exact, then $q(\mathfrak{A})-\operatorname{dim}(\mathcal{A}) \leq k$,

3) $\mathfrak{A}-\operatorname{dim}(\mathcal{B}) \geq \max \{e(\mathfrak{A})-\operatorname{dim}(\mathcal{C}), \quad q(\mathfrak{A})-\operatorname{dim}(\mathcal{A})\}$.

Proof. (1) Since $\mathfrak{A}$ is a contravariantly finite subcategory of $\mathcal{B}$, it follows from Lemma $2.6(1)$ that $e(\mathfrak{A})$ is a contravariantly finite subcategory of $\mathcal{C}$. Since the object $r(\mathcal{C}) \in \mathcal{B}$ and $\mathfrak{A}-\operatorname{dim}(\mathcal{B}) \leq k$, there exists an exact sequence 


$$
0 \rightarrow T_{k} \stackrel{t_{k}}{\longrightarrow} T_{k-1} \rightarrow \cdots \rightarrow T_{1} \stackrel{t_{1}}{\longrightarrow} T_{0} \stackrel{t_{0}}{\longrightarrow} r(\mathrm{C}) \rightarrow 0
$$

with $T_{i}(i=0,1, \cdots, k) \in \mathfrak{A}$ such that the induced sequence

$0 \rightarrow \operatorname{Hom}\left(T, T_{k}\right) \longrightarrow \operatorname{Hom}\left(T, T_{k-1}\right) \rightarrow \cdots \rightarrow \operatorname{Hom}\left(T, T_{1}\right) \longrightarrow \operatorname{Hom}\left(T, T_{0}\right) \longrightarrow \operatorname{Hom}(T, r(C)) \rightarrow 0$

is exact for $T \in \mathfrak{A}$. Applying the exact functor $e: \mathcal{B} \rightarrow \mathcal{C}$ to (2.5), we obtain an exact sequence

$$
0 \rightarrow e\left(T_{k}\right) \stackrel{t_{k}^{*}}{\longrightarrow} e\left(T_{k-1}\right) \rightarrow \cdots \rightarrow e\left(T_{1}\right) \stackrel{t_{1}^{*}}{\longrightarrow} e\left(T_{0}\right) \stackrel{t_{0}^{*}}{\longrightarrow} \mathrm{C} \rightarrow 0
$$

with $e\left(T_{i}\right)(i=0,1, \cdots, k) \in e(\mathfrak{A})$. To prove that $e(\mathfrak{A})-\operatorname{dim}(\mathcal{C}) \leq k$, it is enough to show that the induced sequence

$$
0 \rightarrow \operatorname{Hom}\left(e(T), e\left(T_{k}\right)\right) \rightarrow \cdots \rightarrow \operatorname{Hom}\left(e(T), e\left(T_{0}\right)\right) \rightarrow \operatorname{Hom}(e(T), e r C) \rightarrow 0
$$

is exact. Since the morphisms $T_{0} \rightarrow r(\mathrm{C}), T_{1} \rightarrow \operatorname{Ker}\left(t_{0}\right), \cdots, T_{k} \rightarrow \operatorname{Ker}\left(t_{k-1}\right)$ are right $\mathfrak{A}$-approximations, it follows that the morphisms $e\left(T_{0}\right) \rightarrow e r(C), e T_{1} \rightarrow e\left(\operatorname{Ker}\left(t_{0}\right)\right), \cdots, e\left(T_{k}\right) \rightarrow e\left(\operatorname{Ker}\left(t_{k-1}\right)\right)$ are right $e(\mathfrak{A})$ -approximations. Indeed, the functor $r$ is fully faithful and $r e(\mathfrak{A}) \subseteq \mathfrak{A}$. This implies that the sequence (2.6) is exact. Hence, from Lemma 3.1, we get the inequality $e(\mathfrak{A})-\operatorname{dim}(\mathrm{C}) \leq k$.

(2) Since $\mathfrak{A}$ is a contravariantly finite subcategory of $\mathcal{B}$, it follows from Lemma 2.7 (1) that $q(\mathfrak{A})$ is a contravariantly finite subcategory of $\mathcal{A}$. Let $A$ be an object of $\mathcal{A}$. Since $\mathfrak{A}-\operatorname{dim}(\mathcal{B}) \leq k$, there exists an exact sequence

$$
0 \rightarrow T_{k} \stackrel{s_{k}}{\longrightarrow} T_{k-1} \rightarrow \cdots \rightarrow T_{1} \stackrel{s_{1}}{\longrightarrow} T_{0} \stackrel{s_{0}}{\longrightarrow} i(A) \rightarrow 0
$$

with $T_{i}(i=0,1, \cdots, k) \in \mathfrak{A}$ such that the induced sequence

$$
0 \rightarrow \operatorname{Hom}\left(T, T_{k}\right) \longrightarrow \operatorname{Hom}\left(T, T_{k-1}\right) \rightarrow \cdots \rightarrow \operatorname{Hom}\left(T, T_{1}\right) \longrightarrow \operatorname{Hom}\left(T, T_{0}\right) \longrightarrow \operatorname{Hom}(T, i(A)) \rightarrow 0
$$

is exact for $T \in \mathfrak{A}$. Applying the exact functor $q: \mathcal{B} \rightarrow \mathcal{A}$ to (2.7), we obtain an exact sequence

$$
0 \rightarrow q\left(T_{k}\right) \stackrel{s_{k}^{*}}{\longrightarrow} q\left(T_{k-1}\right) \rightarrow \cdots \rightarrow q\left(T_{1}\right) \stackrel{s_{1}^{*}}{\longrightarrow} q\left(T_{0}\right) \stackrel{s_{0}^{*}}{\longrightarrow} q i(A) \rightarrow 0
$$

with $q\left(T_{i}\right)(i=0,1, \cdots, k) \in q(\mathfrak{A})$. To show that $q(\mathfrak{A})-\operatorname{dim}(\mathcal{A}) \leq k$, it is enough to prove that the sequence

$$
0 \rightarrow \operatorname{Hom}\left(q T, q T_{k}\right) \longrightarrow \operatorname{Hom}\left(q T, q T_{k-1}\right) \rightarrow \cdots \rightarrow \operatorname{Hom}\left(q T, q T_{0}\right) \rightarrow \operatorname{Hom}(q T, q i(A)) \rightarrow 0
$$

is exact. Since the morphisms $T_{0} \rightarrow i(A), T_{1} \rightarrow \operatorname{Ker}\left(s_{0}\right), \cdots, T_{k} \rightarrow \operatorname{Ker}\left(s_{k-1}\right)$ are right $\mathfrak{A}$-approximations, it follows that the morphisms $q\left(T_{0}\right) \rightarrow q i(A), q T_{1} \rightarrow q\left(\operatorname{Ker}\left(s_{0}\right)\right), \cdots, q\left(T_{k}\right) \rightarrow q\left(\operatorname{Ker}\left(s_{k-1}\right)\right)$ are right $q(\mathfrak{A})$-approximations. Indeed, the functor $i$ is fully faithful and $i q(\mathfrak{A}) \subseteq \mathfrak{A}$. This implies that the 
sequence (2.8) is exact. Hence, from Lemma 2.1, we get the inequality $q(\mathfrak{A})-\operatorname{dim}(\mathcal{A}) \leq k$.

The assertion (3) can be easily obtained from the proofs of (1) and (2).

\subsection{Theorem}

Let $(\mathcal{A}, \mathcal{B}, \mathcal{C})$ be a recollement of abelian categories with $\mathcal{B}$ and $\mathcal{C}$ having enough projective objects. If $T$ is a tilting object in $\mathcal{B}$, then there is a tilting torsion pair $(\mathcal{T}(T), \mathcal{F}(T))$ in $\mathcal{B}$.

Furthermore, $\mathcal{T}(T)$ is a contravariantly finite subcategory of $\mathcal{B}$ and $\mathcal{F}(T)$ is a covariantly finite subcategory of $\mathcal{B}$. Suppose in addition that the functor $q=p$ is exact dense, and $e$ is dense, then
(1) $e \mathcal{T}(T)=\mathcal{T}(e T)$;
(2) $q \mathcal{T}(T)=\mathcal{T}(q T)$;
(3) $q \mathcal{F}(T)=\mathcal{F}(q T)$;
(4) $e \mathcal{F}(T)=\mathcal{F}(e T)$.

Proof. For a tilting object $T$, it follows from [11, Proposition 2.1] immediately that $(\mathcal{T}(T), \mathcal{F}(T)$ ) is a tilting torsion pair in $\mathcal{B}$. Since $\operatorname{Hom}(\mathcal{T}(T), \mathcal{F}(T))=0$, we infer that $\mathcal{T}(T)$ is a contravariantly finite and $\mathcal{F}(T)$ is a covariantly finite in $\mathcal{B}$.

To begin with, we verify the first equation $e \mathcal{T}(T)=\mathcal{T}(e T)$. The assertion for the equation (2) is similar. To prove $e \mathcal{T}(T) \subseteq \mathcal{T}(e T)$. For any $X \in e \mathcal{T}(T)$, there is an object $B \in \mathcal{T}(T)$ such that $X=e B$.

So for any short exact sequence $0 \rightarrow B \rightarrow Y^{\prime} \rightarrow T \rightarrow 0$ in $\operatorname{Ext}_{\mathcal{B}}^{1}(T, B)$, which splits such that $Y^{\prime} \cong B \oplus T$. The exact functor $e$ ensures that the sequence $0 \rightarrow e B \rightarrow e Y^{\prime} \rightarrow e T \rightarrow 0$ is exact with $e Y^{\prime} \cong e(B \oplus T)=e B \oplus e T$. Thus, we deduce that $\operatorname{Ext}_{\mathcal{C}}^{1}(e T, e B)=0$. So $X=e B \in \mathcal{T}(e T)$. Conversely, let $X \in \mathcal{T}(e T)$, then there exists an object $B$ in $\mathcal{B}$ such that $X \cong e B$. So we have

$$
\operatorname{Ext}_{\mathcal{C}}^{1}(e T, \quad X) \cong \operatorname{Ext}_{\mathcal{C}}^{1}(e T, \quad e B)=0
$$

Then for any $\delta: 0 \rightarrow B \rightarrow Y^{\prime} \rightarrow T \rightarrow 0$ in $\operatorname{Ext}_{\mathcal{B}}^{1}(T, B)$. Applying the exact functor $e$ we obtain

$$
e \delta: 0 \rightarrow e B \rightarrow e Y^{\prime} \rightarrow e T \rightarrow 0
$$

is in $\operatorname{Ext}_{\mathcal{C}}^{1}(e T, e B)$, which is split. This means that $e Y^{\prime} \cong e B \oplus e T$, but $e B \oplus e T \cong e(B \oplus T)$. So $Y^{\prime} \cong B \oplus T$. Hence $\delta$ is split, thus $\operatorname{Ext}_{\mathcal{B}}^{1}(T, B)=0$. Therefore, $B \in \mathcal{T}(T)$ and $e B \in e \mathcal{T}(T)$. We conclude that $\mathcal{T}(e T) \subseteq e \mathcal{T}(T)$.

Next we shall show the equation $q \mathcal{F}(T)=\mathcal{F}(q T)$, the assertion for the equation (4) is similar. Firstly, for any $Y \in \mathcal{F}(q T)$, we have $\operatorname{Hom}_{\mathcal{A}}(q T, Y)=0$. Since $q$ is dense, it follows that there is an object $Y^{\prime} \in \mathcal{B}$ such that $Y=q Y^{\prime}$. Here we can take $Y^{\prime}=i Y$. Furthermore, the following adjoint isomorphism $\operatorname{Hom}_{\mathcal{B}}(T, i Y) \cong \operatorname{Hom}_{\mathcal{A}}(q T, Y)$ yields that $i Y \in \mathcal{F}(T)$. And the equivalence $\operatorname{Id}_{\mathcal{A}} \simeq q i$ implies that $Y \simeq q(i Y) \in q \mathcal{F}(T)$. Hence this proves that $\mathcal{F}(q T) \subseteq q \mathcal{F}(T)$. Conversely, let $Q \in q \mathcal{F}(T)$, then there exists an object $M$ in $\mathcal{B}$ such that $M \in \mathcal{F}(T)$, that is, $q M=Q$. It is enough to prove that $q M \in \mathcal{F}(q T)$. It follows from the adjoint pair $(i, q=p)$ that $\operatorname{Hom}_{\mathcal{A}}(q T, q M) \cong \operatorname{Hom}_{\mathcal{B}}(i q T, M)$

On the other hand, from [4, proposition 2.6] we have the following exact sequence in $\mathcal{B}$

$$
0 \rightarrow \operatorname{Ker} \mu_{T} \rightarrow l e T \rightarrow T \stackrel{\lambda_{T}}{\longrightarrow} i q T \rightarrow 0 .
$$


Set $K=\operatorname{Ker} \lambda_{T}$, where $\lambda_{T}$ is the unit of the adjoint pair $(q, i)$, then there is a short exact sequence

$$
0 \rightarrow K \rightarrow T \rightarrow i q T \rightarrow 0
$$

Applying the functor $\operatorname{Hom}_{\mathcal{B}}(-, M)$ to the above sequence, we obtain that

$$
0 \rightarrow \operatorname{Hom}_{\mathcal{B}}(i q T, M) \rightarrow \operatorname{Hom}_{\mathcal{B}}(T, M) \rightarrow \operatorname{Hom}_{\mathcal{B}}(K, M) \rightarrow \cdots
$$

For $M \in \mathcal{F}(T)$, we have $\operatorname{Hom}_{\mathcal{B}}(T, M)=0$. Hence, $\operatorname{Hom}_{\mathcal{B}}(i q T, M)=0$. So, $\operatorname{Hom}_{\mathcal{A}}(q T, q M)=0$, which shows that $q M=Q \in \mathcal{F}(q T)$. Therefore, $q \mathcal{F}(T) \subseteq \mathcal{F}(q T)$. Finally, we conclude that $q \mathcal{F}(T)=\mathcal{F}(q T)$.

\subsection{Corollary}

Let $(\mathcal{A}, \mathcal{B}, \mathcal{C})$ be a recollement of abelian categories with the exact functor $q$, and assume that $T$ is a tilting object in $\mathcal{B}$, then $\mathcal{T}(T)-\operatorname{dim}(\mathcal{B}) \geq \max \{e(\mathcal{T}(T))-\operatorname{dim}(\mathcal{C}), \quad q(\mathcal{T}(T))-\operatorname{dim}(\mathcal{A})\}$.

\section{Conclusions}

This paper treats interesting questions on recollements of abelian categories. By investigating how contravariantly finite subcategories behaves in a recollement $(\mathcal{A}, \mathcal{B}, \mathcal{C})$ between abelian categories, we obtain that if $\mathfrak{A}-\operatorname{dim}(\mathcal{B}) \leq k$ and $r e(\mathfrak{A}) \subseteq \mathfrak{A}, i q(\mathfrak{A}) \subseteq \mathfrak{A}$, Then

$$
\mathfrak{A}-\operatorname{dim}(\mathcal{B}) \geq \max \{e(\mathfrak{A})-\operatorname{dim}(\mathcal{C}), \quad q(\mathfrak{A})-\operatorname{dim}(\mathcal{A})\}
$$

As an application, for a tilting object $T$ in $\mathcal{B}$, we also get an upper bound of relative dimensions with respect to tilting objects $\mathcal{T}(T)-\operatorname{dim}(\mathcal{B}) \geq \max \{e(\mathcal{T}(T))-\operatorname{dim}(\mathcal{C}), \quad q(\mathcal{T}(T))-\operatorname{dim}(\mathcal{A})\}$.

\section{Acknowledgment}

This work was supported by National Natural Science Foundation of China (Grant No. 11271119).

\section{References}

[1] Beilinson, A., Bernstein, J., \& Deligne, P. (1982). Faisceaux pervers. Analysis and Topology on Singular Spaces, Asterisque, 100, 5-171.

[2] Angeleri, H. L., Koenig, S., \& Liu, Q. (2011). Recollements and tilting objects. J Pure Appl Algebra, 215, 420-438.

[3] Psaroudakis, C., \& Vitória, J. (2014). Recollements of module categories. Appl Categor Struct, 22, 579-593.

[4] Psaroudakis, C. (2014). Homological theory of recollements of abelian categories. J Algebra, 398, 63-110.

[5] Dugas, A. S. (2007). Representation dimension as a relative homological invariant of stable equivalence. Algebr Represent Theor., 10, 223-240.

[6] Auslander, M., \& Solberg, 0. (1993). Relative homology and representation theory I. Comm. Algebra, 21(9), 2995-3031. 
[7] Auslander, M., \& Reiten, I. (1991). Applications of homologically finite subcategories. Adv. in Math., 86, 111-152.

[8] Rotman, J. J. (2008). An Introduction to Homological Algebra. 2nd edition. Springer.

[9] Auslander, M., Reiten, I., \& Smalø, S. O. (1997). Representation Theory of Artin Algebras, Cambridge Studies in Advanced Mathematics.

[10] Smalø, S. (1991). Functional finite subcategories over triangular matrix rings. Proceedings of the American Mathematical Society (pp. 651-656).

[11] Happel, D., \& Unger, L. (2011). Representation dimension and tilting. J Pure Appl Algebra, 215, 2315-2327.

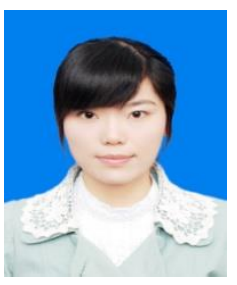

Xuerong Fu received a master's degree from College of Mathematical Sciences, Qufu Normal University, Shandong, P. R. China in 2014. She is now pursuing the Ph. D degree in College of Applied Sciences, Beijing University of Technology, Beijing, P. R. China. Her research interests include representation theory of algebras and coalgebras, homological algebra, module theory, approximation theory and algebraic topology in pure mathematics.

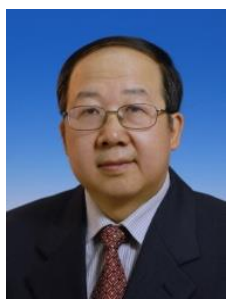

Hailou Yao works as a full professor, PhD advisor, and the dean for Department of Pure Mathematics in College of Applied Sciences, Beijing University of Technology. He received a $\mathrm{Ph}$. D degree in department of mathematics, Beijing Normal University, Beijing, P. R. China. And he visited the University of Sherbrooke, Canada, from April to July in 1999 and from June to November in 2001. His research interests include representation theory of algebras and coalgebras, homological algebra, module theory, the theory of ordered system, matrix theory, approximation theory and algebraic topology in pure mathematics. 